\title{
New results on covers and partial spreads of polar spaces
}

go back

full screen

close

quit
Andreas Klein
Klaus Metsch

\begin{abstract}
We investigate blocking sets of projective spaces that are contained in cones over quadrics of rank two. As an application we obtain new results on partial ovoids, partial spreads, and blocking sets of polar spaces. One of the results is that a partial ovoid of $H\left(3, q^{2}\right)$ with more than $q^{3}-q+1$ points is contained in an ovoid. We also give a new proof of the result that a partial spread of $Q(4, q)$ with more than $q^{2}-q+1$ lines is contained in a spread; this is the first common proof for even and odd $q$. Finally, we improve the lower bound on the size of a smallest blocking set of the symplectic polar space $W(3, q), q$ odd.
\end{abstract}

Keywords : polar spaces, partial spreads, partial ovoids, blocking sets, covers MSC 2000: 05B25, 51E12, 51E20, 51E21

\section{Introduction}

Let $H\left(3, q^{2}\right)$ be a hermitian surface of $\mathrm{PG}\left(3, q^{2}\right)$. The lines it contains are called its generators. An ovoid of $H\left(3, q^{2}\right)$ is a set of points of $H\left(3, q^{2}\right)$ meeting every generator exactly once, and a partial ovoid is a set of points meeting every generator in at most one point. It is known that $H\left(3, q^{2}\right)$ has ovoids, for example a hermitian curve $H\left(2, q^{2}\right)$ that is obtained by intersecting $H\left(3, q^{2}\right)$ with a nontangent hyperplane. A blocking set of $H\left(3, q^{2}\right)$ is a set of points that meets every generator in at least one point. The same definition is used for all other polar spaces of rank two, that is polar spaces that contain lines but no planes.

The origin of the paper was the problem of finding the largest partial ovoid of $H\left(3, q^{2}\right)$ that is not an ovoid. This problem we learned from Gary Ebert [4]. It is a simple calculation to see that an ovoid of $H\left(3, q^{2}\right)$ has exactly $q^{3}+1$ points,

$$
\text { is a simple calculation to see that an ovoid of } H\left(3, q^{2}\right) \text { has exactly } q^{3}+1 \text { points, }
$$





\section{Blocking sets contained in quadrics}

If one studies partial spreads or covers of quadrics, then the set of points of the quadric that are not covered (for spreads) respectively the set of points of the quadric that are covered more than once (for covers) have similar properties. It is extremely useful to study the intersection of these sets with tangent hyperplanes. We shall do this in the case $Q^{-}(5, q)$. The crucial observation is in the following lemma.

full screen

close

Lemma 2.1. Consider in $\mathrm{PG}(4, q)$ a quadric that is a cone with vertex a point $P$ over a non-degenerate elliptic quadric $Q^{-}(3, q)$. Suppose that $B$ is a set of at most $2 q$ points contained in the quadric. If every solid of $\mathrm{PG}(4, q)$ meets $B$, then one of the following occurs:

(a) Some line of the quadric is contained in $B$.

(b) $|B|>\frac{9}{5} q+1, P \in B$, and there exists a unique line $l$ of the quadric that meets $B$ in at least $1+\frac{1}{3}|B|$ points. This line has at most $|B|-1-q$ points in $B$.

Proof. Denote by $l_{i}, i=1, \ldots, q^{2}+1$, the lines of the quadric on $P$. If $P$ is not in $B$, then we use that each line $l_{i}$ lies on a solid meeting the quadric only in $l_{i}$ to deduce that $|B| \geq q^{2}+1$; but $|B| \leq 2 q$, so $P \in B$. Put $b:=|B|-1$ and $b_{i}:=\left|l_{i} \cap B\right|-1$. Let $\mathcal{S}$ be the set consisting of the $q^{4}$ solids that do not contain $P$, and put $b_{S}:=|B \cap S|$ for $S \in \mathcal{S}$. Since all solids $S$ meet $B$, then $b_{S} \geq 1$ for all $S \in \mathcal{S}$.

If one of the lines $l_{i}$ is contained in $B$, there is nothing to show. We may thus assume that this is not the case. Consider different lines $l_{i}$ and $l_{j}$ and choose points $R_{i} \in l_{i}$ and $R_{j} \in l_{j}$ such that $R_{i}, R_{j} \notin B$. Then there are $q^{2}$ solids in $\mathcal{S}$ containing the line $R_{i} R_{j}$, and every point of $B$ not lying on $l_{i} \cup l_{j}$ appears in exactly $q$ of these. Thus, if we sum up $b_{S}$ for the $q^{2}$ solids $S$ of $\mathcal{S}$ on the line $R_{i} R_{j}$, then we obtain $\left(b-b_{i}-b_{j}\right) q$. As $b_{S} \geq 1$ for all $S \in \mathcal{S}$, it follows that $b-b_{i}-b_{j} \geq q$, that is

$$
b_{i}+b_{j} \leq b-q \text { for } i \neq j .
$$

We use for integers $x \geq 1$ the inequality

$$
1 \leq x-\frac{5}{6}\left(\begin{array}{l}
x \\
2
\end{array}\right)+\frac{1}{2}\left(\begin{array}{l}
x \\
3
\end{array}\right)
$$

to obtain

$$
q^{4}=|\mathcal{S}| \leq \sum_{S \in \mathcal{S}}\left(b_{S}-\frac{5}{6}\left(\begin{array}{c}
b_{S} \\
2
\end{array}\right)+\frac{1}{2}\left(\begin{array}{c}
b_{S} \\
3
\end{array}\right)\right) .
$$




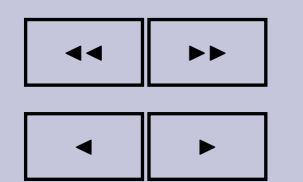

page $4 / 16$

go back

full screen

close

quit

For the terms on the right hand side, standard counting arguments give

$$
\begin{aligned}
\sum_{S \in \mathcal{S}} b_{S} & =\sum_{i} b_{i} q^{3}=b q^{3}, \\
\sum_{S \in \mathcal{S}}\left(\begin{array}{c}
b_{S} \\
2
\end{array}\right) & =\sum_{i<j} b_{i} b_{j} q^{2}=\frac{1}{2} q^{2} b^{2}-\frac{1}{2} q^{2} \sum_{i} b_{i}^{2}, \text { and } \\
\sum_{S \in \mathcal{S}}\left(\begin{array}{c}
b_{S} \\
3
\end{array}\right) & =\sum_{i<j<k} b_{i} b_{j} b_{k} q=\frac{1}{6} b^{3} q-\frac{1}{2} b q \sum_{i} b_{i}^{2}+\frac{1}{3} q \sum_{i} b_{i}^{3} .
\end{aligned}
$$

Putting this together and dividing by $q$ results in

$$
q^{3} \leq b q^{2}-\frac{5}{12} b^{2} q+\frac{1}{12} b^{3}+\frac{1}{12} \sum_{i} b_{i}^{2}\left(5 q-3 b+2 b_{i}\right) .
$$

CASE 1: $b_{i} \leq \frac{1}{2}(b-q)$ for all $i$. Then

$$
\sum_{i} b_{i}^{2}\left(5 q-3 b+2 b_{i}\right) \leq \sum_{i} b_{i} \frac{1}{2}(b-q)(4 q-2 b)=b(b-q)(2 q-b) \text {. }
$$

Combining this with (4) gives $0 \leq \frac{1}{6} q(2 q-b)(b-3 q)$. As $b=|B|-1 \leq 2 q-1$, this is a contradiction.

CASE 2: $\max \left\{b_{i}\right\}>\frac{1}{2}(b-q)$. We may assume that $b_{1}=\max \left\{b_{i}\right\}$. From (1) we obtain $b_{i} \leq b-q-b_{1}$ for $i \geq 2$. As $\sum b_{i}=b$, it follows that

$$
\begin{aligned}
\sum_{i} b_{i}^{2}\left(5 q-3 b+2 b_{i}\right) & \leq b_{1}^{2}\left(5 q-3 b+2 b_{1}\right)+\sum_{i \geq 2} b_{i}\left(b-q-b_{1}\right)\left(3 q-b-2 b_{1}\right) \\
& =b_{1}^{2}\left(5 q-3 b+2 b_{1}\right)+\left(b-b_{1}\right)\left(b-q-b_{1}\right)\left(3 q-b-2 b_{1}\right) .
\end{aligned}
$$

Combining this with (4) multiplied by 12 , we find that $0 \leq f\left(b_{1}\right)$, where $f \in \mathbb{Z}[x]$ is defined by

$$
f:=q\left(9 b q-12 q^{2}-b^{2}+6 x^{2}-5 b x+3 x q\right) .
$$

As $f$ is a polynomial of degree two in $x$ and since

$$
f\left(\frac{b}{3}\right)=f\left(\frac{b-q}{2}\right)=q(2 q-b)(b-3 q) / 6<0
$$

it follows that $f(x)<0$ for $x$ between $\frac{1}{2}(b-q)$ and $\frac{1}{3} b$; note that $b<2 q$ implies that $\frac{1}{3} b>\frac{1}{2}(b-q)$. As $b_{1}>\frac{1}{2}(b-q)$ and $f\left(b_{1}\right) \geq 0$, it follows that $b_{1}>\frac{b}{3}$. Thus $\left|l_{1} \cap B\right|>1+\frac{b}{3}$ and hence $\left|l_{1} \cap B\right| \geq 1+\frac{1}{3}|B|$ (since $|B|=b+1$ ). From (1) we also have $b_{1}<b-q$, that is $\left|l_{1} \cap B\right|<|B|-q$ and hence $\left|l_{1} \cap B\right| \leq|B|-q-1$. 




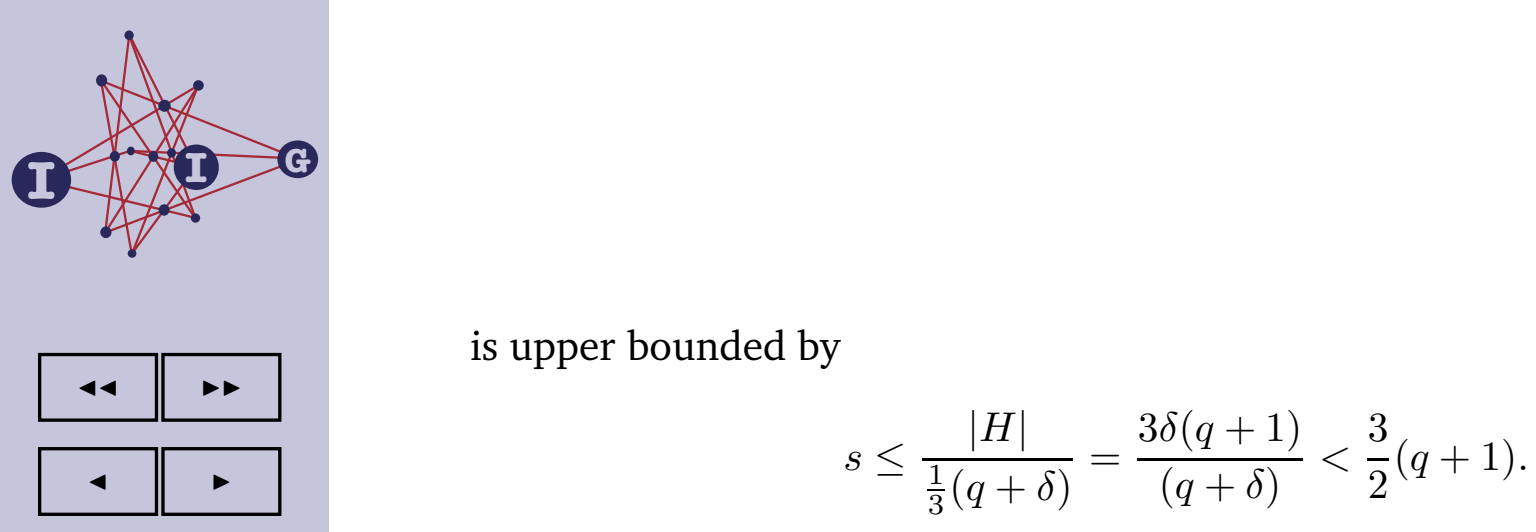

page $7 / 16$

go back

full screen

close

quit

Also, as $\left|l_{i} \cap H\right|<\delta$ and $|H|=\delta(q+1)$, then $s \geq q+2$. Finally we remark that $\delta \leq q-1$ and the hypothesis $1+\frac{1}{3}(q+\delta) \leq\left|l_{i} \cap H\right| \leq \delta-1$ for all $i$ imply that $q \geq 8$.

PART 3 . We shall show in this part that every hyperplane that contains two of the lines $l_{i}$ contains at least $\frac{1}{2}(q+1)$ of the lines $l_{i}$. For this, suppose that $X$ is a hyperplane that contains exactly $c \geq 2$ lines $l_{i}$, say $l_{1}, \ldots, l_{c}$. We may assume that $\left|l_{1} \cap H\right| \geq\left|l_{i} \cap H\right|$ for $i=1, \ldots, c$.

Consider the hyperbolic solid $\left\langle l_{1}, l_{2}\right\rangle$, and let $R$ be a point of $l_{1}$ that is not a hole. As at least two points of $l_{2}$ are not holes, we find a non-hole $R^{\prime}$ on $l_{2}$ such that $R R^{\prime}$ is a secant line to the quadric. Then the line $R R^{\prime}$ has no hole. Since the line $R R^{\prime}$ lies on $q^{2}$ planes that are contained in $X$ but not in $\left\langle l_{1}, l_{2}\right\rangle$ and since the number of holes is $\delta(q+1)<q^{2}$, we find a plane $\pi$ on $R R^{\prime}$ that is contained in $X$ but not in $\left\langle l_{1}, l_{2}\right\rangle$ and that has no hole.

Put $|X \cap H|=r q+\delta$. By Part 1, every solid of $X$ meets $H$ in at least $r$ points. Considering the $q+1$ solids of $X$ on $\pi$ taking into account that two of these contain $l_{1}$ resp. $l_{2}$, we find that

$$
\begin{aligned}
r q+\delta & \geq(q-1) r+\left|l_{1} \cap H\right|+\left|l_{2} \cap H\right| . \\
\Rightarrow \quad r+\delta & \geq\left|l_{1} \cap H\right|+\left|l_{2} \cap H\right| .
\end{aligned}
$$

Each of the lines $l_{i}$ with $i>c$ meets $X$ in a unique point, which might be in $H$. This implies that

$$
r q+\delta=|X \cap H| \leq \sum_{i \leq c}\left|l_{i} \cap H\right|+s-c \leq c\left|l_{1} \cap H\right|+s-c .
$$

Writing $r q+\delta=q(r+\delta)-\delta q+\delta$, we find

$$
\left|l_{1} \cap H\right| q+\left|l_{2} \cap H\right| q-\delta q+\delta \leq c\left|l_{1} \cap H\right|+s-c .
$$

Assume that $c \leq \frac{q}{2}$. Using $\left|l_{i} \cap H\right| \geq \frac{1}{3}(q+\delta)$ and $s<\frac{3}{2}(q+1)$, it follows that

$$
\frac{3}{2} q \cdot \frac{q+\delta}{3}<\delta q-\delta+q+\frac{3}{2}
$$

As $\delta \leq q-1$, this leads to $q<5$. But we have seen in Part 2 that $q \geq 8$. This contradiction shows that $c \geq \frac{1}{2}(q+1)$.

PART 4. Here we study the case that every solid that contains two of the lines $l_{i}$ contains at least $\frac{1}{2}(q+1)$ of the lines $l_{i}$. Consider a solid $S$ containing $u \geq 2$ 



\section{Covers of $Q^{-}(5, q)$ and $Q(4, q)$}

The technique of the previous section can be slightly modified to be applicable to covers. We shall demonstrate this for $Q^{-}(5, q)$. However, we start more generally with weighted line sets that cover all points.

Lemma 4.1. Suppose that $w$ is a function from the set $\mathcal{L}$ of lines of $Q^{-}(5, q)$ to $\mathbb{Z}$. For every point $P$ denote by $w_{P}+1$ the sum of the values $w(l)$ running over all lines $l$ on $P$. Suppose that $w_{P} \geq 0$ for all $P$.

If $\delta:=\sum_{l \in \mathcal{L}} w(l)-\left(q^{3}+1\right) \leq 1+\frac{4}{5} q$, then there exist (not necessarily distinct) lines $l_{1}, \ldots, l_{\delta}$ of $Q^{-}(5, q)$ with the following property: For every point $P$, the number $w_{P}$ is equal to the number of lines $l_{i}$ that pass through $P$.

Proof. We have $\sum_{l \in \mathcal{L}} w(l)=q^{3}+1+\delta$ and thus

$$
\sum_{P \in Q^{-}(5, q)}\left(w_{P}+1\right)=\left(q^{3}+1+\delta\right)(q+1) \Rightarrow \sum_{P \in Q^{-}(5, q)} w_{P}=\delta(q+1) .
$$

Hence $\delta \geq 0$ with equality if and only if $w_{P}=0$ for all points $P$ of $Q^{-}(5, q)$. Thus, the theorem is correct in the case $\delta=0$. Suppose now that $0<\delta \leq$ $\frac{4}{5} q+1$. Embed $Q^{-}(5, q)$ in a natural way in $\operatorname{PG}(5, q)$. For every subset $A$ of $\mathrm{PG}(5, q)$, denote by $w(A)$ the sum of the $w_{P}$ for $P \in A \cap Q^{-}(5, q)$. Notice that $w(\mathrm{PG}(5, q))=\delta(q+1)$.

As every hyperplane of $\operatorname{PG}(5, q)$ meets $Q^{-}(5, q)$ in 1 modulo $q$ points and since $\sum w(l)=q^{3}+1+\delta$, then $w(H)$ is congruent to $\delta$ modulo $q$ for every hyperplane $H$ of $\operatorname{PG}(5, q)$. As a matter of fact, when $S$ is a solid with $w(S)=0$, then $w(\operatorname{PG}(5, q))=\delta(q+1)$ implies that $w(H)=\delta$ for every hyperplane $H$ on $S$. In other words:

(*) $w(H)>\delta$ for a hyperplane $H$ implies $w(S)>0$ for all solids $S$ of $H$.

Put $c:=\min \left\{w_{P} \mid w_{P}>0\right\}$, and denote by $P$ a point satisfying $w_{P}=c$. Then the sum of the $w(l)$ for the lines $l$ of $Q^{-}(5, q)$ not on $P$ is $q^{3}+\delta-c$. As the tangent hyperplane $P^{\perp}$ has $\left(q^{2}+1\right) q+1$ points in $Q^{-}(5, q)$, it follows that

$$
w\left(P^{\perp}\right)=(c+1)(q+1)+q^{3}+\delta-c-\left(q^{2}+1\right) q-1=c q+\delta .
$$

Put $B:=\left\{X \in P^{\perp} \mid w_{X}>0\right\}$. Then $w(B)=c q+\delta$. As $w(X) \geq c$ for all $X \in B$, this implies that $|B| \leq(c q+\delta) / c \leq q+\delta$. From $(*)$ we see that all solids of $P^{\perp}$ meet $B$. As $\delta \leq 1+\frac{4}{5} q$, then Lemma 2.1 implies that $B$ contains a line $l_{0}$. Define a new function $w^{\prime}$ from the lines of $Q^{-}(5, q)$ to $\mathbb{Z}$ with $w^{\prime}(l)=w(l)$ for $l \neq l_{0}$, and $w^{\prime}\left(l_{0}\right):=w\left(l_{0}\right)-1$. As $w(P) \geq 1$ for all $P \in l_{0}$, we see that $w^{\prime}$ fulfills the hypothesis of the lemma. As $\sum w^{\prime}(l)$ is one less than $\sum w(l)$, an inductive argument completes the proof. 




